\title{
Quadrupedal dinosaurs did not evolve fully pronated forearms: new evidence from the ulna
}

\author{
Joel D. Hutson \\ Acta Palaeontologica Polonica 60 (3), 2015: 599-610 doi:http://dx.doi.org/10.4202/app.00063.2014
}

Therians (marsupials and placentals), archosaurs, and chameleons are remarkable in that they evolved postures and gaits with inturned forelimbs. However, recent studies have indirectly recognized that, unlike fully pronated therian and chameleon forearms, dinosaur forearms were mechanically constrained by semi-pronated (misaligned) joints. This has led to the hypothesis that quadrupedal dinosaurs mitigated this constraint via proximal migration of the radius, indirectly forming a more pronated, tubular manus distally. To test this hypothesis, a standardized pose was used to examine the forearm pronation of ornithischian dinosaurs that were obligatory quadrupeds and facultative bipeds. Results show that only restructuring of the distal, not the proximal radius, causes additional pronation of the pre-axial edge of the carpus, but also unexpectedly reveal that the ulna may help form a tubular manus by supinating the post-axial edge. Thus, relative to the plane of the elbow joint the wrist and finger joints remain wholly semi-pronated. These findings do not support the hypothesis that a tubular cross-section evolved in dinosaurs to pronate the manus further to allow the finger joints to participate in locomotion. Instead these results indicate that quadrupedal dinosaurs tended to abandon propulsive use of their wrist and finger joints by converting their carpus + metacarpus into a vertical stilt-like extension of the forearm. Prior studies have overlooked that this divergent path to parasagittal forelimb kinematics had its phylogenetic basis in the retention of the semi-pronated forearm joint alignment that is plesiomorphic to tetrapods. Thus, this test provides the first functional explanation for the convergent responses of quadrupedal archosaurs to their misaligned forearm joints, and provides a foundation for elucidating why the quadrupedal evolution of archosaur forelimbs diverged from those of therians and chameleons.

Key words: Archosauria, Dinosauria, Theria, Chamaeleonidae, locomotion, posture, functional morphology, biomechanics.

Joel D. Hutson [jhutson@elgin.edu], Department of Biological Sciences, Northern Illinois University, 1425 West Lincoln Highway, DeKalb, Illinois, 60115-2825, USA; present address: Department of Biology, Elgin Community College, 1700 Spartan Drive, Elgin, Illinois, 60123-7193, USA. 
This is an open-access article distributed under the terms of the Creative Commons

Attribution License (for details please see creativecommons.org), which permits unrestricted use, distribution, and reproduction in any medium, provided the original author and source are credited.

\author{
FoF \\ Far) Supplementary file $(110.1 \mathrm{kB})$
}

